\title{
Obstrução por hérnia diafragmática oculta estrangulada: relato de caso
}

\author{
Obstruction by strangulated occult diaphragmatic hernia: case report
}

Obstruido por hernia diafragmática oculta estrangulada: reporte de caso

Marcos André Filgueiras Dias ${ }^{1 *}$, Messias Froes da Silva Júnior², Saullo Anderson Costa Monteiro1, Marcel de Aguiar Raposo da Câmara Coelho', Gabriel Reis Di Tommaso', Juan Eduardo Rios Rodriguez ${ }^{3}$, Mirella Cruz Lira ${ }^{3}$, Rubem Alves da Silva Júnior ${ }^{1}$.

\section{RESUMO}

Objetivo: Relatar caso de abdome agudo obstrutivo por estrangulamento de hérnia diafragmática oculta. Relato do caso: Paciente do sexo masculino, com quadro de abdome agudo obstrutivo, transferido do interior do estado e recebido, após cerca de quatro dias de evolução, em hospital da capital, com dor toracoabdominal intensa, distensão abdominal importante e parada da eliminação de flatos e fezes. Radiografia mostrava formação heterogênea mal definida em tórax. Tomografia computadorizada evidenciou conteúdo intestinal em base de hemitórax esquerdo, associado à distensão exagerada do cólon. Indicada laparotomia exploradora, com redução e correção da hérnia diafragmática com sucesso. Considerações finais: O quadro clínico da hérnia diafragmática varia consideravelmente, dependente da biomecânica do trauma, estado fisiológico e individualidade anatômica do paciente, estágio de evolução, tamanho da hérnia, tempo decorrido do trauma, os quais também determinam sua morbimortalidade. É importante que o diagnóstico e o tratamento sejam realizados de modo precoce a fim de evitar complicações tardias, a exemplo da obstrução e infarto de alça intestinal por estrangulamento.

Palavras-chave: Abdome agudo, Cirurgia geral, Diafragma, Hérnia, Tomografia.

\begin{abstract}
Objective: To report a case of acute obstructive abdomen due to strangulation of occult diaphragmatic hernia. Case report: Male patient, with acute obstructive abdomen, transferred from the interior of the state and received, after about four days of evolution, in a hospital in the capital, with severe thoracoabdominal pain, significant abdominal distension and stopping the elimination of flatus and feces. Radiography showed poorly defined heterogeneous chest formation. Computed tomography showed intestinal content based on the left hemithorax, associated with exaggerated colon distension. Exploratory laparotomy was indicated, with successful reduction and correction of the diaphragmatic hernia. Final considerations: The clinical picture of diaphragmatic hernia varies considerably, depending on the biomechanics of the trauma, the physiological state and anatomical individuality of the patient, stage of evolution, size of the hernia, time elapsed from the trauma, which also determine its morbidity and mortality. It is important that the diagnosis and treatment are performed early in order to avoid late complications, such as obstruction and bowel loop infarction due to strangulation.
\end{abstract}

Keywords: Acute abdomen, General surgery, Diaphragm, Hernia, Tomography.

\footnotetext{
${ }^{1}$ Hospital Universitário Getúlio Vargas (HUGV), Manaus-AM, Brasil.

*E-mail: medkinho@gmail.com

2 Instituto de Cirurgia do Estado do Amazonas (ICEA), Manaus-AM, Brasil.

${ }^{3}$ Universidade Federal do Amazonas (UFAM), Manaus-AM, Brasil.
} 


\section{RESUMEN}

Objetivo: Reportar un caso de abdomen obstructivo agudo debido a estrangulamiento de hernia diafragmática oculta. Informe del caso: Paciente masculino, con abdomen obstructivo agudo, transferido desde el interior del estado y recibido, después de unos cuatro días de evolución, en un hospital de la capital, con dolor toracoabdominal intenso, distensión abdominal significativa y detención de la eliminación de flatos y heces. La radiografía mostró una formación torácica heterogénea mal definida. La tomografía computarizada mostró contenido intestinal basado en el hemitórax izquierdo, asociado con una distensión exagerada del colon. Se indicó laparotomía exploratoria, con reducción y corrección exitosa de la hernia diafragmática. Consideraciones finales: El cuadro clínico de la hernia diafragmática varía considerablemente, dependiendo de la biomecánica del trauma, el estado fisiológico y la individualidad anatómica del paciente, la etapa de evolución, el tamaño de la hernia, el tiempo transcurrido desde el trauma, lo que también determina su morbilidad y mortalidad. Es importante que el diagnóstico y el tratamiento se realicen temprano para evitar complicaciones tardías, como la obstrucción y el infarto de asa intestinal debido al estrangulamiento.

Palabras clave: Abdomen agudo, Cirugía general, Diafragma, Hernia, Tomografía.

\section{INTRODUÇÃO}

Pode-se caracterizar a hérnia diafragmática como o resultado de uma injúria no músculo diafragma, com a ruptura da barreira física que esse músculo proporciona entre as cavidades torácica e abdominal (WILLIAMS M, et al., 2019).

Essa injúria pode ser de etiologia congênita, geralmente presente nos pacientes pediátricos, ou adquirida, nos pacientes adultos, pela maior exposição a situações traumáticas (SOLDO I, et al., 2017). Dentre as causas adquiridas, temos o trauma toracoabdominal fechado, como nos acidentes automobilísticos, e o trauma penetrante, por armas brancas ou de fogo (PEREIRA JÚNIOR GA, 2001).

Os sinais e sintomas muitas vezes podem ser discretos, inespecíficos e não condizentes com a situação clínica do paciente, tendo em vista os poucos sintomas que relatam (COSTA GBG, et al., 2019). O quadro clínico não necessariamente está relacionado com o tamanho da herniação, que tende a aumentar com o tempo, pela própria fisiologia da região em estudo (BEDI RS e SINGH HJ, 1991; ERCAN M, et al., 2016; ALLAN Z, et al., 2017). A apresentação clínica pode não ocorrer até a gravidez ou trabalho de parto, quando há aumento considerável da pressão intra-abdominal (PEREIRA JÚNIOR GA, 2001).

De fato, pode haver variadas apresentações clínicas dos quadros de hérnias diafragmáticas, desde sua fase aguda até a crônica, sendo, portanto, de grande importância o diagnóstico e tratamento precoce, a fim de evitar complicações (GRIMES OF, 1974; WILLIAMS M, et al., 2019).

Nos pacientes jovens com obstrução do cólon, a presença de hérnia diafragmática crônica é fortemente sugerida no diagnóstico diferencial nos quadros de abdome agudo obstrutivo, como também naqueles com história de trauma toracoabdominal penetrante (PEREIRA JÚNIOR GA, 2001).

Em relação ao diagnóstico, quando precoce, em grande parte é feito cirurgicamente, durante laparotomia exploradora. Já quando é realizado tardiamente, é resultado da exacerbação de sintomas respiratórios ou de obstrução do trato intestinal de pacientes previamente expostos a traumas toracoabdominais (GRIMES OF, 1974; LENOT B, et al., 1990; ZHAO H, et al., 2017). Neste caso, exames de imagem complementares, como radiografia, tomografia computadorizada (TC), avaliação focada com a ultrassonografia no trauma (FAST), ressonância magnética, podem ser usados (ZHAO H, et al., 2017; WILLIAMS M, et al., 2019).

Em até $67 \%$ dos casos, os exames de imagem podem ser normais, de modo que uma história admissional bem colhida e um exame físico, detalhado e cuidadoso, são de extrema relevância (FELICIANO DV, et al., 1988; SHACKLETON KL, et al., 1998). Além dos exames inalterados, também pode ocorrer, durante a exploração cirúrgica, o achado de algum elemento anatômico que não foi mostrado nos exames de imagem e que também pode ser lesado com a evolução natural do aumento do conteúdo herniado, como, por exemplo, a presença de um baço acessório (DA COSTA KG, et al., 2019). 
O tratamento consiste na correção cirúrgica com a sutura do diafragma, quer o diagnóstico pré-operatório de hérnia diafragmática seja agudo ou crônico, com base nos exames de imagem complementares ou ainda, nos casos duvidosos, na indicação cirúrgica urgente (PEREIRA JÚNIOR GA, 2001). Este relato tem por objetivo fomentar a atividade da propedêutica médica com informações essenciais para o diagnóstico claro e ágil das hérnias diafragmáticas ocultas em adultos, norteando a indicação precisa do seu tratamento cirúrgico definitivo, precocemente, nos diferentes níveis de atendimento.

\section{DETALHAMENTO DO CASO}

Paciente do sexo masculino, 33 anos, agricultor, natural e procedente da zona rural de um município do interior do estado do Amazonas, previamente hígido. Histórico de agressão física nesta localidade, em 2015, que resultou em ferimentos múltiplos na região anterior da base do hemitórax esquerdo, pela utilização de gargalo de garrafa. Informa que procurou atendimento médico local, contudo, não foi evidenciado comprometimento ventilatório, optando-se por conduta conservadora, síntese dos ferimentos e curativos. Permaneceu em observação por cerca de dois dias e recebeu alta.

Nos anos subsequentes, negou ter apresentado queixas, mantendo-se ativo em suas atividades laborais. Em maio de 2019, procurou novamente atendimento médico em sua localidade, agora por quadro de abdome agudo obstrutivo e foi referenciado para avaliação cirúrgica na capital do estado, com sinais vitais estáveis. No hospital da capital, foi admitido pela equipe de cirurgia geral, relatando, há cerca de quatro dias, dor no hemitórax esquerdo, dor e distensão abdominais intensas, dispneia, hiporexia, náuseas, vômitos e parada da eliminação de flatos e fezes. Negou cirurgias prévias e outras comorbidades.

Durante a avaliação cirúrgica: eutrófico, dispneico, com 24 incursões respiratórias por minuto, frêmito tóraco-vocal reduzido e murmúrio vesicular abolido em base de hemitórax esquerdo, oximetria de pulso de $98 \%$ ao ar ambiente, algo desidratado, normocorado, ritmo cardíaco regular, frequência cardíaca de 100 batimentos por minuto, normotenso, abdome distendido, sem equimoses, hematomas ou circulação colateral, ruídos hidroaéreos (RHA) pouco aumentados, peristaltismo de luta presente, hiper-timpânico, defesa abdominal, sem irritação peritoneal franca, espaço de Traube hiper-timpânico e sinal de Piparote negativo.

Na radiografia de tórax, realizada no primeiro atendimento, havia uma formação heterogênea na base do hemitórax esquerdo, sem definição da cúpula diafragmática esquerda, com gás de permeio, que podia corresponder a conteúdo intestinal. Para melhor definição da hipótese diagnóstica, após a avaliação, o paciente foi submetido à TC de tórax e abdome, no hospital.

Os achados tomográficos sugeriam conteúdo abdominal, de provável origem cólica, domiciliado na porção inferior do hemitórax esquerdo, repleto de níveis hidroaéreos, em íntimo contato com a pleura parietal ânterolateral, atelectasia do lobo pulmonar inferior esquerdo, sutil deslocamento do mediastino para direita, áreas de pneumatose intestinal e borramento grosseiro da parede da víscera oca estrangulada (Figura 1A), derrame pleural pequeno à direita e moderado à esquerda, além de imagem sugestiva da falha da cúpula diafragmática esquerda, de colo estreito, com cerca de $3 \mathrm{~cm}$ de diâmetro (Figura 1B).

Figura 1 - Tomografia helicoidal computadorizada de tórax caracterizando o conteúdo herniado à esquerda.

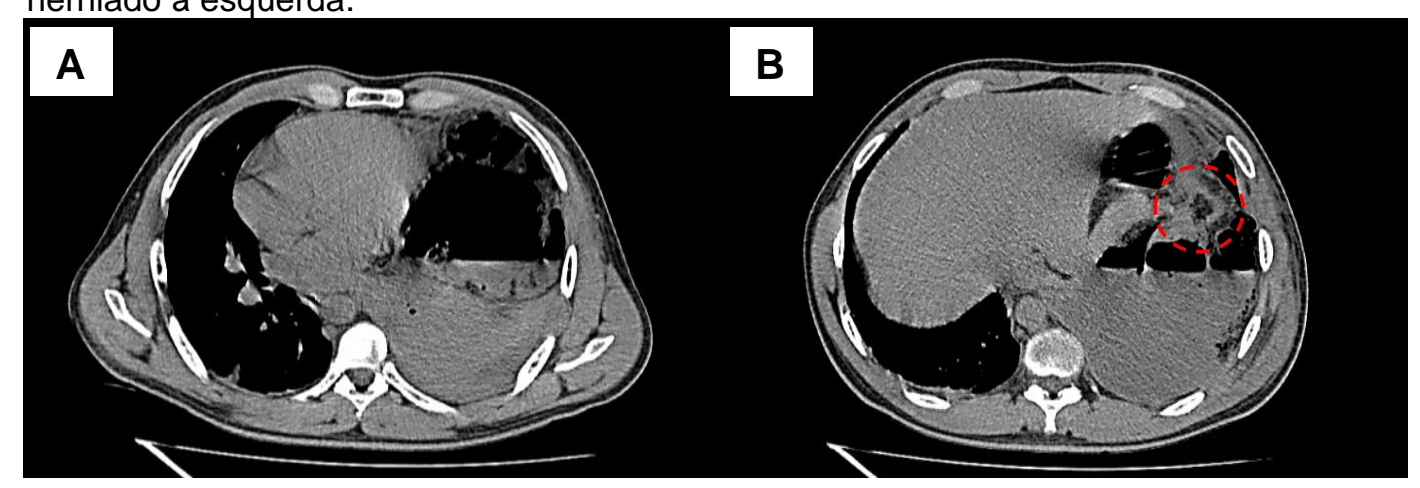

Legenda: (A) Distensão do conteúdo abdominal na cavidade pleural; (B) Falha muscular diafragmática, em destaque. Fonte: Dias MAF, et al., 2020. 
Ainda na TC, observou-se uma generosa distensão cólica a montante da flexura esplênica, com início na topografia do hipocôndrio esquerdo (Figura 2A) e mais evidente no ceco (Figura 2B), sem este padrão em cólon descendente e sigmóide, além de discreta distensão e edema da parede do intestino delgado, principalmente, no íleo terminal. A câmara gástrica parcialmente colabada e com resíduo exíguo (Figura 2A), discreta quantidade de líquido livre na cavidade abdominal, ausência de gás na ampola retal, arcabouço costal sem evidências de distorções, não podendo descartar áreas focais de pneumoperitôneo.

Figura 2 - Tomografia helicoidal computadorizada de abdome caracterizando a distensão cólica.

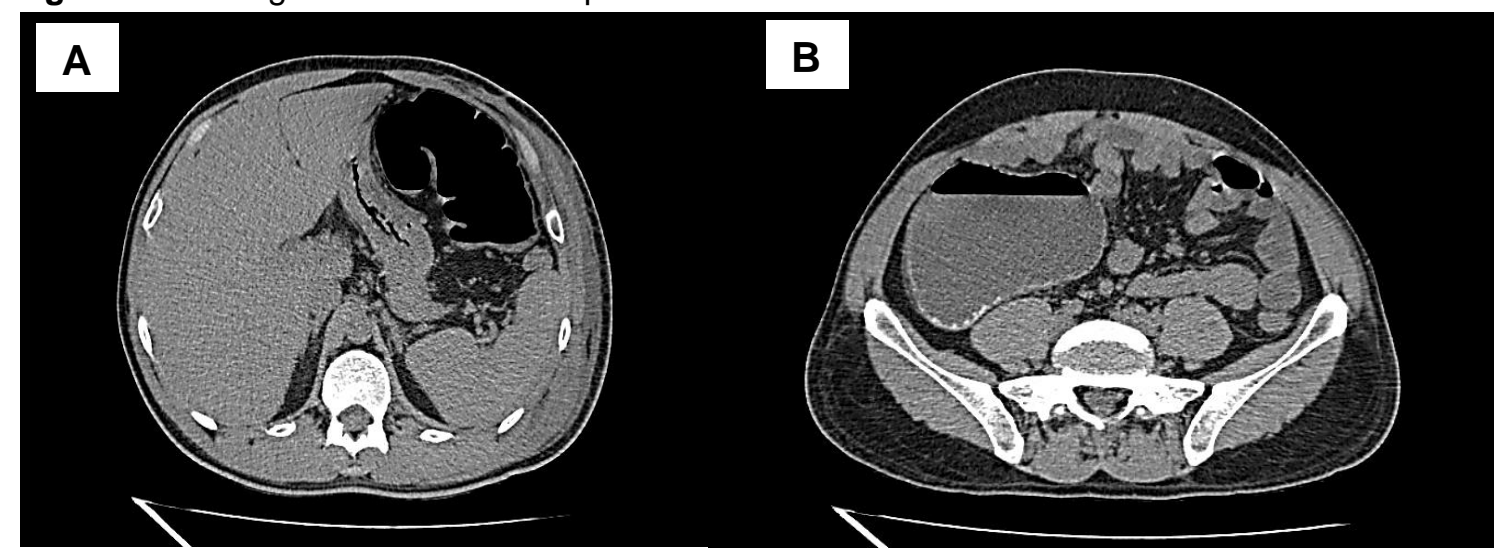

Legenda: (A) Início da distensão cólica, a montante da flexura esplênica; (B) Distensão exagerada do ceco.

Fonte: Dias MAF, et al., 2020.

Com a melhor definição diagnóstica, foi indicada a laparotomia exploradora de urgência para correção da hérnia diafragmática esquerda estrangulada e instituída cobertura antibiótica. O paciente, após ser orientado, assinou o termo de consentimento e foi conduzido ao centro cirúrgico. No inventário da cavidade, citamos: flexura esplênica do cólon (transverso distal e descendente proximal) e epíplon estrangulados por falha de 4 cm na cúpula diafragmática esquerda; generosa distensão do cólon a montante desta flexura até o ceco; laceração da tênia anti-mesocólica no ceco; discreta distensão do íleo e pouco líquido livre.

No ato cirúrgico, foi realizada a liberação dos cólons à Cattell-Braasch e Mattox, optando-se por apendicectomia tática para descompressão do grande volume do cólon, com tratamento do coto apendicular à Parker-Kerr e cecorrafia contínua em plano único sobre a tênia anti-mesocólica. A frenotomia de cada lado da falha permitiu melhor redução do conteúdo e debridamento do lobo pulmonar inferior esquerdo. Houve rompimento da parede da flexura e extravasamento de conteúdo luminal na cavidade herniária, prontamente aspirado, com irrigação exaustiva da cavidade pleural com soro fisiológico morno.

Após a frenorrafia e confecção da drenagem fechada de tórax (DFT), foi procedida a colectomia segmentar da região da flexura esplênica, preservando os vasos cólicos médios e esquerdos, com fechamento do coto do cólon esquerdo em dois planos, seguido de irrigação abundante da cavidade abdominal com soro fisiológico morno. Após a síntese da aponeurose abdominal, foi finalizada a maturação primária da transversostomia à esquerda. O diagnóstico pós-cirúrgico contemplou hérnia diafragmática estrangulada esquerda com necrose do cólon transverso distal e descendente proximal, além do epíplon.

Depois da intervenção cirúrgica, o paciente foi conduzido ao centro de tratamento intensivo, onde permaneceu por 48 horas sem intercorrências, sendo transferido para a enfermaria cirúrgica masculina. Em relação aos comemorativos da prescrição médica, além da analgesia em dose plena e outros sintomáticos, foi mantida a antibioticoterapia, solicitados cuidados com dreno de tórax e colostomia, com quantificação e caracterização do débito de ambos, fisioterapia respiratória, troca diária de curativos, elevação da cabeceira entre 30 e 45 graus, além de estímulo precoce à deambulação.

No pós-operatório recente, não apresentou ílio adinâmico prolongado e a colostomia funcionou plenamente (Figura 3A). Porém, conforme controle tomográfico de tórax realizado, o paciente evoluiu com 
empiema no hemitórax esquerdo, atelectasia e encarceramento pulmonar do lobo inferior esquerdo, derrame pleural residual à direita, mas com mediastino já centrado (Figura 3B). Esses achados justificaram uma toracotomia esquerda secundária no $25^{\circ}$ dia de pós-operatório (DPO), para decorticação pulmonar e limpeza da cavidade pleural, transcorrida sem intercorrências, com retorno à enfermaria cirúrgica masculina.

Figura 3 - Contraste da boa evolução no 6ำ DPO com a associação de complicações pulmonares.

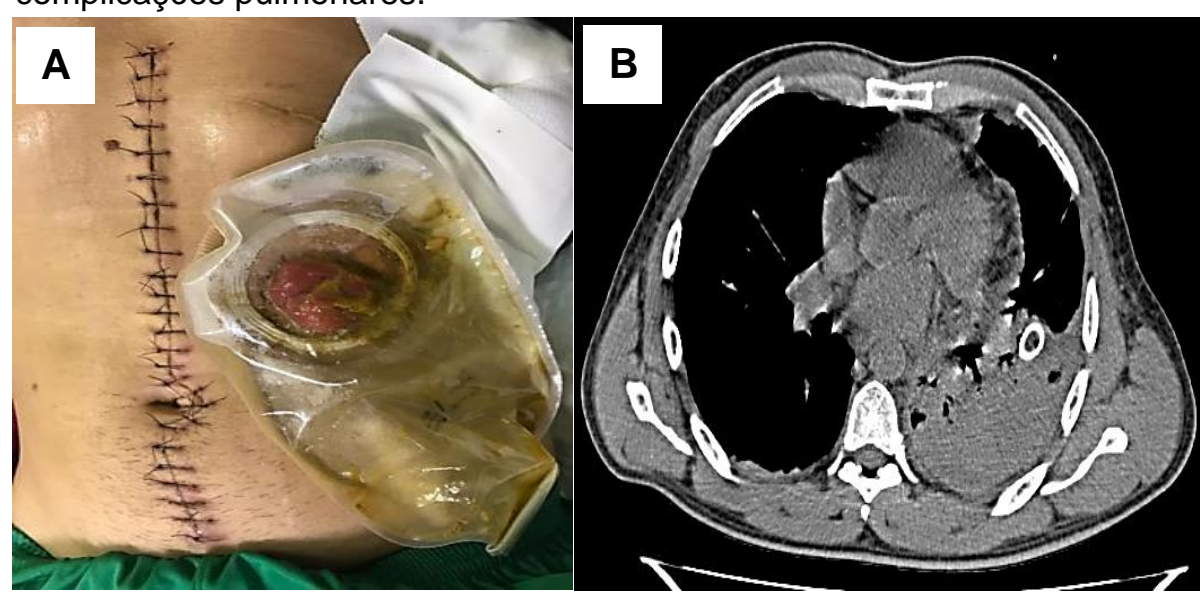

Legenda: (A) Colostomia funcionante; (B) Atelectasia, encarceramento pulmonar e empiema pleural à esquerda. Fonte: Dias MAF, et al., 2020.

O paciente alcançou melhora satisfatória precoce após a segunda intervenção cirúrgica, recebendo alta no $29^{\circ}$ DPO, sendo orientado e encaminhado para seguimento ambulatorial com as especialidades da cirurgia torácica e do aparelho digestivo. Por volta do $6^{\circ}$ mês de pós-operatório (MPO), já apresentava excelente cicatrização das feridas (Figuras 4A e 4B). Atualmente, no 10 MPO, com colostomia, ainda está impossibilitado para suas atividades laborais, apesar do bom estado geral, aguardando a liberação pela cirurgia torácica para realização da cirurgia de reconstrução do trânsito intestinal.

Figura 4 - Evolução pós-operatória da cicatrização das feridas no 6 MPO.

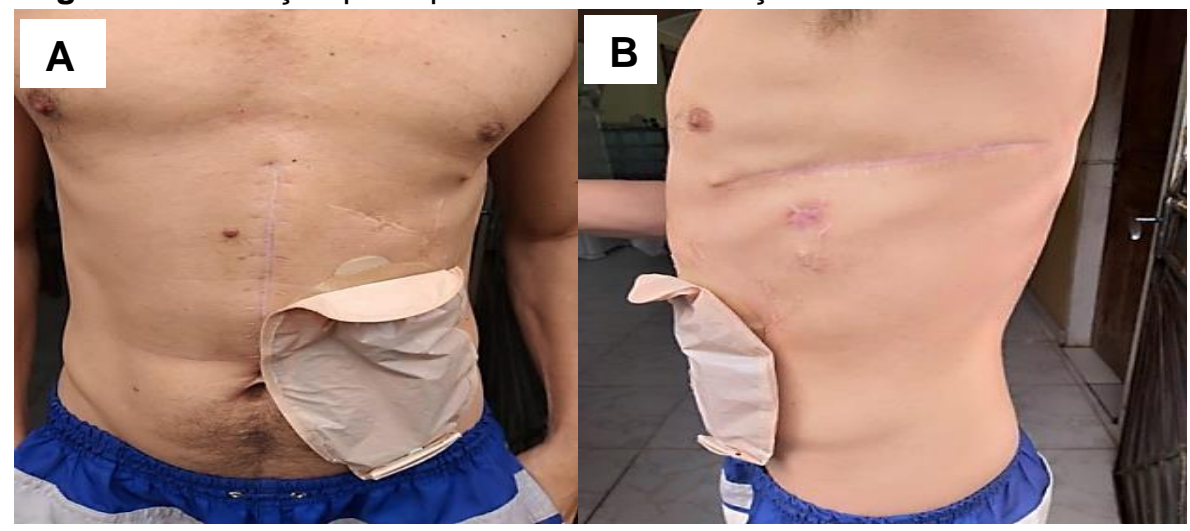

Legenda: (A) Cicatrizes prévias e da laparotomia mediana; (B) Cicatrizes da toracotomia secundária e da DFT. Fonte: Dias MAF, et al., 2020.

\section{DISCUSSÃO}

Dentre as alterações no exame físico passíveis de serem observadas em quadros de estrangulamento de hérnia diafragmática, destacam-se: diminuição do murmúrio vesicular em $90 \%$ dos casos, taquipneia em $66,7 \%$ e RHA em hemitórax esquerdo em cerca de 52,4\% dos pacientes, além de desconforto torácico, vômitos, dor e distensão abdominais (GAINE FA, et al., 2013). No detalhamento deste caso, foi possível contemplar os dois achados mais frequentes ao exame clínico, corroborando a literatura. Os RHA no hemitórax estavam abolidos pela lesão avançada da víscera oca. 
Uma progressão na apresentação dos sinais e sintomas é descrita em fases. A primeira é aguda, tendo início com o trauma e término com a recuperação das outras lesões associadas. A segunda é a fase latente e ocorre quando há o contato do conteúdo abdominal com estruturas torácicas, podendo levar horas a semanas após a lesão. Por fim, a terceira é a fase obstrutiva, onde meses a anos após a lesão podem surgir sinais e sintomas de obstrução, isquemia e estrangulamento (GRIMES OF, 1974). Por exemplo, este paciente iniciou a manifestação dos sintomas obstrutivos quatro anos após o trauma torácico prévio.

Nessa última fase, o doente pode apresentar dispneia significativa ou estrangulamento intratorácico de órgão abdominal e o diagnóstico tardio contribui para o aumento da morbimortalidade (LENOT B, et al., 1990; WILLIAMS M, et al., 2019). Como constatado neste caso, as hérnias diafragmáticas geralmente levam a sintomas agudos no decorrer do tempo, uma vez que a pressão negativa na cavidade torácica aumenta a lesão inicial no diafragma e o volume herniário na cavidade torácica, por um mecanismo de sucção das alças e outros órgãos (BEDI RS e SINGH HJ, 1991; ERCAN M, et al., 2016; CORBELLINI C, et al., 2017).

Com isso, a maior parte das hérnias diafragmáticas tende a aumentar de tamanho com o passar do tempo, na sua evolução natural (ALLAN Z, et al., 2017), o que pode acabar levando a complicações catastróficas, conforme exposto no delineamento deste trabalho, explicando, em parte, o aparecimento súbito do quadro de abdome agudo obstrutivo. Todavia, de modo controverso, há casos relatados de pacientes que evoluíram com regressão espontânea da hérnia diafragmática, evidenciando como a particularidade de cada indivíduo se faz presente (CAVALCANTE GH, et al., 2019).

$\mathrm{Na}$ investigação por imagem, o método com menor custo, boa acessibilidade e tradicionalmente utilizado é a radiografia simples de tórax, uma vez que é eficiente em detectar lesões diafragmáticas em pelo menos $40 \%$ dos casos, com maior acurácia para lesões do lado esquerdo (SILVA GP, et al., 2018), como também, alças em tórax (MORGAN B, et al., 2010). Único método de imagem disponível na unidade de saúde onde o paciente foi atendimento inicialmente, no interior do estado, contribuiu como subsídio para elaboração da hipótese diagnóstica primária e para nortear a propedêutica durante a avaliação especializada, na capital.

Entretanto, os mecanismos dos achados da lesão diafragmática podem sugerir a necessidade de exames complementares adicionais, como a TC de tórax e abdome, que evidencia em até $57,1 \%$ dos casos, alterações sugestivas de lesão diafragmática e herniação (LIM BL, et al., 2017; WILLIAMS M, et al., 2019).

No presente caso, a TC disponível no hospital foi de fundamental valia para o adequado esclarecimento, não só da presença da hérnia diafragmática esquerda, como também, para a identificação das demais lesões associadas já descritas aqui anteriormente.

A evolução com sinais e sintomas de obstrução e infarto de alça intestinal aumenta consideravelmente a mortalidade. Tal mortalidade, por apresentação tardia da lesão diafragmática, para o paciente com encarceramento do conteúdo gastrointestinal é de aproximadamente $20 \%$, aumentando para até $57 \%$ quando há estrangulamento intestinal (SULLIVAN RE, 1966). Neste relato, o paciente apresentou uma complicação tardia da hérnia diafragmática oculta, e, diante de todo o exposto, evoluiu, além dos achados já citados, com complicações pós-operatórias inerentes, aumentando a morbimortalidade do seu quadro.

Na região Amazônica, particularmente, o acesso terrestre conta com uma malha limitada de estradas, uma parte de difícil tráfego, transformando o acesso fluvial numa alternativa amplamente factível e necessária, mas que é refém do regime das águas na época da vazante dos rios, tornando-se também inviável em determinado período do ano. Dessa forma, o acesso aéreo é a única alternativa em muitos casos, mas que também esbarra na limitação do funcionamento dos pequenos aeródromos das localidades, nos fatores climáticos adversos para o voo, sem falar no maior custo deste tipo de transporte nessa região.

Por compreender uma vasta área geográfica, além da já noticiada escassez de atendimento médico especializado no interior dos estados amazônicos, muitos pacientes só conseguem assistência médica adequada quando os sintomas estão exacerbados e não há mais fatores de melhora. Isso acarreta transferências médicas, muitas vezes em caráter urgente para a capital, justificadas por um melhor esclarecimento diagnóstico e necessidade de avaliação especializada. Isso resulta, não raro, em viagens aéreas onerosas ou fluviais prolongadas, demorando até vários dias, dependendo da localidade de origem. 
Diante do exposto, é imprescindível ao atendimento médico generalista ou durante avaliação especializada de urgência, que uma história admissional seja bem colhida e um exame físico consistente seja realizado, de modo a nortear o profissional no diagnóstico e na conduta adequados. Em inúmeras localidades afastadas das capitais amazônicas, por exemplo, os exames de imagem complementares, como TC e até mesmo radiografia simples de tórax, são escassos e, por vezes, inexistentes, sendo papel do médico, tratar o doente com os recursos que the estão disponíveis. Desse modo, relatos como esse colaboram com a formação médica e auxiliam na ênfase da deteç̧ão de sinais e sintomas precoces da hérnia diafragmática, a fim de proporcionar um melhor prognóstico para o paciente.

\section{REFERÊNCIAS}

1. ALLAN Z, et al. Traumatic diaphragmatic rupture with underlying lung laceration and tension pneumoperitoneum. Journal of Surgical Case Reports, 2017;2017(6):1-3.

2. BEDI RS e SINGH HJ. Delayed presentation of traumatic diaphragmatic hernia. The Indian Journal of Chest Diseases and Allied Sciences, 1991;33(3):139-41.

3. CAVALCANTE GH, et al. Redução espontânea de hérnia diafragmática traumática: Relato de caso. Revista de Patologia do Tocantins, 2019;6(3):30-3.

4. CORBELLINI C, et al. Diaphragmatic rupture: a single-institution experience and literature review. Ulus Travma Acil Cerrahi Derg, 2017;23(5):421-6.

5. COSTA GBG, et al. Hérnia diafragmática oculta após trauma - Relato de caso. Revista Eletrônica Acervo Saúde, 2019;22(e663):1-6.

6. DA COSTA KG, et al. Delayed diaphragmatic hernia after open trauma with unusual content: Case report. International Journal of Surgery Case Reports, 2019;64(1):50-3.

7. ERCAN M, et al. Dual mesh repair for a large diaphragmatic hernia defect: An unusual case report. International Journal of Surgery Case Reports, 2016;28(1):266-9.

8. FELICIANO DV, et al. Delayed diagnosis of injuries to the diaphragm after penetrating wounds. The Journal of Trauma: Injury, Infection, and Critical Care, 1988;28(8):1135-44.

9. GAINE FA, et al. The etiology, associated injuries and clinical presentation of post traumatic diaphragmatic hernia. Bulletin Emergency and Trauma, 2013;1(2):76-80.

10. GRIMES OF. Traumatic injuries of the diaphragm. Diaphragmatic hernia. The American Journal of Surgery, $1974 ; 128(2): 175-81$.

11. LENOT B, et al. Les ruptures du diaphragme de révélation tardive. Annales de Chirurgie Thoracique Cardio-vasculaire, 1990;44(2):157-60.

12. LIM BL, et al. Traumatic diaphragmatic injuries: a retrospective review of a 12-year experience at a tertiary trauma centre. Singapore Medical Journal, 2017;58(10):595-600.

13. MORGAN B, et al. Traumatic diaphragmatic injury. Journal of Royal Army Medical Corps / BMJ Military Health, 2010;156(3):139-44.

14. PEREIRA JÚNIOR GA. Hérnia diafragmática traumática. Revista do Colégio Brasileiro de Cirurgiões, 2001;28(5):37582.

15. SHACKLETON KL, et al. Traumatic diaphragmatic injuries: spectrum of radiographic findings. RadioGraphics, 1998;18(1):49-59.

16. SILVA GP, et al. Thoracotomy compared to laparotomy in the traumatic diaphragmatic hernia. Systematic review and proportional methanalysis. Acta Cirurgica Brasileira, 2018;33(1):49-66.

17. SOLDO I, et al. Laparoscopic treatment of morgagni hernia: Report of three cases. Acta Clinica Croatica, 2017;56(2):318-22.

18. SULLIVAN RE. Strangulation and obstruction in diaphragmatic hernia due to direct trauma. Report of two cases and review of the english literature. The Journal of Thoracic and Cardiovascular Surgery, 1966;52(5):725-34.

19. WILLIAMS M, et al. Recognition and management of diaphragmatic injury in adults. UpToDate, 2019;1-33.

20. ZHAO H, et al. Delayed traumatic parasternal hernia causes jejunal necrosis: A case report. Trauma Case Reports, 2017;9(1):30-3. 